\title{
Mastopexia de aumento, técnica de quinta generación
}

\section{Augmentation mastopexy, fifth generation technique}

\author{
Navarro, R.*, Torreblanca, L.**, Enríquez, A.***
}

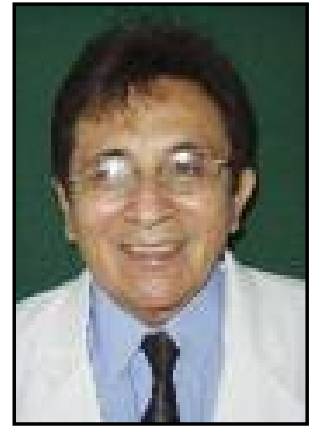

Navarro, $\mathbf{R}$.

\section{Resumen}

Presentamos una nueva técnica de mastopexia, más sencilla, y que evita recidivas además de disminuir el riesgo de carcinoma mamario. El estudio se realiza sobre 25 pacientes femeninas de 25 a 50 años de edad, con ptosis mamaria. Con las pacientes de pie se realizó el marcaje tradicional; si las mamas son pequeñas se colocan implantes en el plano submuscular, y si son grandes, en el retroglandular. Para evitar recidivas se colocaron dos tirantes internos con suturas irreabsorbibles. Si el complejo resultante glándula-implante es grande y pesado, se fija un tirante de la mama al periostio hemiclavicular y otro al periostio de la tercera costilla; si es pequeño o mediano, únicamente se fija a la costilla. Realizamos controles entre 1 mes y 3 años tras la intervención. Conseguimos corregir ptosis de entre 5 a $12 \mathrm{~cm}$., sin recidiva en ninguno de los controles, ni pseudo-ptosis postoperatoria. El ejercicio físico fue posible a los 15 días de la cirugía con el apoyo de un sujetador elástico. La cicatrización fue altamente satisfactoria. Las únicas complicaciones presentadas fueron dehiscencias parciales en 2 pacientes y sensación de ardor, tirantez y elongación de la areola en pacientes cuya ptosis excedía los $12 \mathrm{~cm}$ y con areolas grandes. La facilidad de ejecución de la técnica y su seguridad, permiten que la cirugía sea ambulatoria. Proponemos esta técnica, que denominamos de quinta generación, que evita la cicatriz periareolar, la inframamaria, la vertical y la "T" invertida; además, elimina parte de ambos cuadrantes superiores de la mama, lo que disminuye notablemente la posibilidad de desarrollar ulteriores casos de carcinoma mamario y evita recidivas con el uso del tirante clavicular.

\author{
Palabras clave Mastopexia. Aumento mamario. Técni- \\ ca quirúrgica. \\ Código numérico 5210-52103
}

\begin{abstract}
We present a new mastopexy technique, simpler, which precludes the recidivism and reduces the risk of breast cancer. The study was based on 25 female patients between 25 to 50 years old with breast ptosis. With the patients standing up, was realized the traditional marks. If breasts were small, implants were positioned by submuscular way, and if bigger, the way was retroglandular. For recidivism prevention, it was positioned 2 internal straps; if the gland-implant was bigger and heavier an immobile strap from the breast to the clavicle periostium and to the 3rd rib, was realized; if it would result smaller or median size only were fixed to the third ribs with a dermal flap. Controls were realized between 1 month to 3 years. Ptosis was corrected between 5 to $12 \mathrm{~cm}$ without any recidivism, neither post surgery pseudo-ptosis. With the support of an elastic brassier, physical exercise is recommended after 15 days. The scarring was highly satisfactory. Complications were partial disruptions in 2 patients and ardors, tautness sensation and areolar enlargement in patients with more than $12 \mathrm{~cm}$ ptosis and wide areola. Because of the execution simplicity and security, surgery was ambulatory. We propose this technique called 5th generation that precludes the periareolar, the inframamary, the vertical and the T scar; partial elimination of both upper breasts quadrants lessen the possibility of breast cancer in this upper area and ptosis recidivism by the use of the internal straps.
\end{abstract}

\begin{tabular}{|ll}
\hline Key words & $\begin{array}{l}\text { Mastopexy. Mammary augmentation. } \\
\text { Surgical Technique. }\end{array}$ \\
Numeral Code & $5210-52103$
\end{tabular}




\section{Introducción}

La mastopexia se encuentra entre las cirugías mas solicitadas por mujeres de 25 a 50 años con vida sexual activa. Existe más de una clasificación de ptosis mamaria, sin embargo, la medición más efectiva de diagnostico es el descenso de los pezones más allá de la línea transversal imaginaria que une el punto medio de cada brazo.

La corrección de la ptosis no se logra únicamente con la colocación de prótesis mamarias, sino que suele requerir otro procedimiento complementario. El error más frecuente es tratar de corregir la ptosis con la colocación de implantes más grandes de lo adecuado, dando como resultado una exaltación del defecto y una aparente obesidad de la paciente, así como la recidiva de la patología.

Los objetivos de la cirugía de Mastopexia son: 1) Lograr una mayor turgencia a las mamas, 2) Disminuir el diámetro transversal de ambas mamas, 3) Producir una proyección antero-superior de la mama en vez de volumen, 4) Disminuir tamaño y peso, y sobre todo, 5) Colocar el pezón en su posición ideal y sin recidiva. Por todo esto, es importante que cuando se requiera la colocación de un implante, éste sea del diámetro más pequeño posible.

La mastopexia ha tenido a lo largo de su existencia continuas mejoras, dando como resultado 4 generaciones definidas. La primera (Fig. 1 A) fue encabezada por Weisenberger (1), quien propuso la mamoplastia de reducción como elemento útil en la mastopexia, al igual que hicieron Thorek (2), Passot (3), Strombeck (4), Aufricht (5) y Noel (6). Posteriormente Mckissok (7), Goulián (8), Perras (9) y Paulette Regnault (10) constituyeron la segunda generación (Fig. 1 B), con la utilización de incisiones periareolar y en “ $T$ " invertida inferior. La tercera generación (Fig. 1 C) se caracterizó por la práctica de incisión periareolar única y longitudinal inferior, aportadas por Hinderer (11), Arié (12) y Pitanguy (13) y su posterior simplificación por Peixoto (14). Finalmente, la cuarta generación (Fig. 1D), marcada por la técnica periareolar actual de Ribeiro (15), Pontes (16), Fellicio (17), Benelli (18) y Sampaio (19).

Como precursora de esta técnica citaremos la denominada «media luna creciente superior» (Fig. 1 E), aportada por Orlando (20), Rappaport (21), Pucket (22), Gulyas (23), Kirwan (24), Karnes (25), Graf (26), Ceydeli (27), Auersvald (28), Cárdenas - Camarena y Ramírez Macias (29), De La Fuente y Martín Del Hierro (30), Frey (31), Gasperoni (32), Holmstrom (33), Lewis (34), Weiner (35), Andrews (36), Bartels (37), Auclair (38), Brink (39), Kuzbari (40) y Marconi (41).

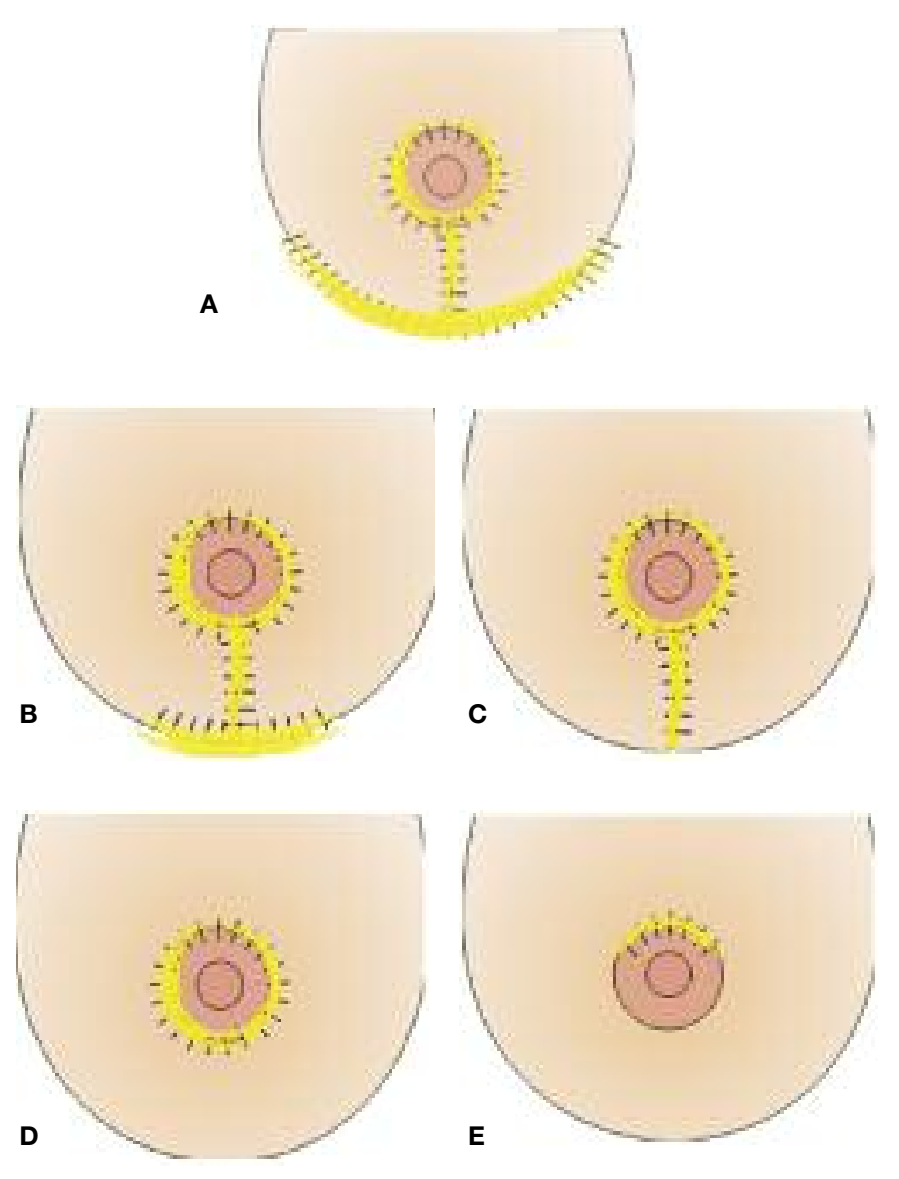

Fig. 1. A) Primera Etapa 1914-1945. B) Segunda Etapa 1945-1980. C) Tercera Etapa 1980-2000. D) Cuarta Etapa 2000-2004: solo de sustento, sin elongación ni fijación costal. E) Quinta Etapa 2004-en adelante: media luna creciente elongada con fijación costal.

La técnica que nosotros presentamos, que se diferencia de la anterior porque elongamos y fijamos la media luna creciente a la costilla, permite por su facilidad de ejecución, simplicidad, rapidez, seguridad con respecto a la innervación y vascularización del complejo areola pezón y versatilidad, ser realizada incluso por cirujanos en entrenamiento, no únicamente en mastopexias, sino también para corrección de mamas tuberosas, asimétricas e hipertróficas (cuando éstas son virginales de tipo glandular estrogénico no graso). Permite también evitar el uso de materiales sintéticos, como mallas de Marlex y lo más importante, no presenta recidiva a lo largo de los años. Reduce a su vez el riesgo de carcinoma mamario al extirpar casi en un $80 \%$ ambos cuadrantes superiores mamarios, donde radican el $48 \%$ de los carcinomas in situ sobre todo en la edad de nuestras pacientes, por lo que en pacientes con antecedentes heredo-familiares de carcinoma mamario que quisieran someterse a una mastopexia, podría ser recomendable. Finalmente, pensamos que con la correcta disección de la cavidad receptora, es posible evitar el problema de pseudopto- 

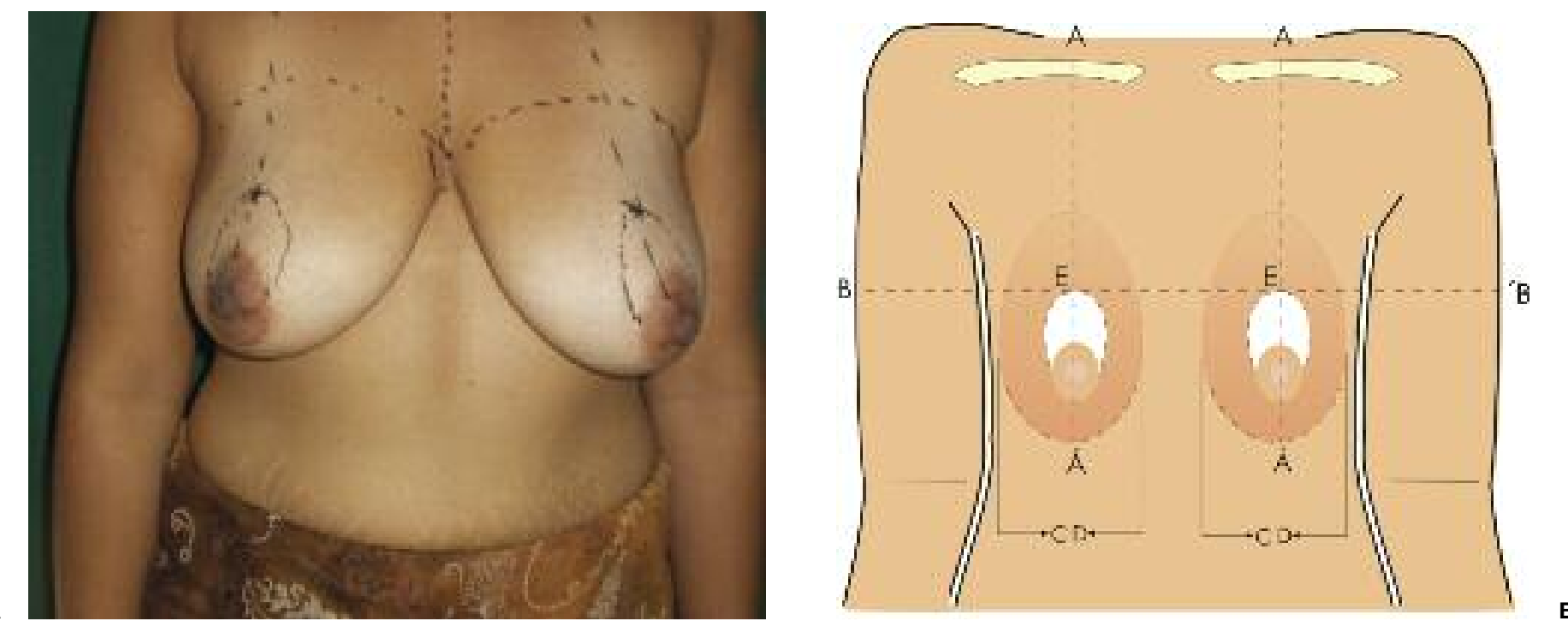

Fig. 2. A) Paciente en bipedestación con marcaje preoperatorio. B) a.-Líneas medio-claviculares. b.-Mitad transversa de ambos brazos. c-d.Anchura de la mama. e.-Encrucijada de líneas (futura posición de la areola).

sis postoperatoria ("bottoming-out"). Además, cuando el colgajo dérmico resulta corto, podemos elongar la media luna creciente mediante una zeta-plastia que asegure el riego vascular.
Material y Método

Operamos 25 pacientes de sexo femenino entre los años 2004 y 2007, de edades comprendidas en un rango de entre 25 y 50 años y una media de edad de 38 años, 2 de ellas nulíparas (Tabla I). Antes de la

Tabla. I.

\begin{tabular}{|c|c|c|c|c|c|c|c|c|c|c|c|}
\hline $\begin{array}{l}\text { Paciente } \\
\text { Edad } \\
\text { Paridad }\end{array}$ & $\begin{array}{l}\text { Elev } \\
\text { Dcha. } \\
\mathrm{cm} .\end{array}$ & $\begin{array}{c}\text { Elev } \\
\text { Izq. } \\
\mathrm{cm}\end{array}$ & $\begin{array}{l}\text { Alt. } \\
\text { Asoc. }\end{array}$ & $\begin{array}{c}\text { Dist } \\
\text { CAP } \\
\text { SSM } \\
\text { Pre. cm }\end{array}$ & $\begin{array}{l}\text { Vol. } \\
\text { Imp. } \\
\text { En } \\
\text { cc }\end{array}$ & $\begin{array}{c}\text { Tejido } \\
\text { Resecado } \\
\text { en grs. }\end{array}$ & $\mathrm{VC}$ & SU & SD & $\begin{array}{c}\text { Dist } \\
\text { CAP } \\
\text { SSM } \\
\text { Post. cm }\end{array}$ & C.C. \\
\hline $1-26 \mathrm{M}$ & 6 & 7 & No & 7 & 260 & $100-220$ & SG & $\mathrm{Si}$ & No & 7 & No \\
\hline 2- $33 \mathrm{M}$ & 10 & 12 & $\mathrm{Si}$ & 8 & 240 & $250-300$ & $\mathrm{SG}$ & No & $\mathrm{Si}$ & 7 & $\mathrm{Si}$ \\
\hline 3- $50 \mathrm{M}$ & 7 & 8 & No & 7 & 280 & $100-150$ & $\mathrm{RM}$ & No & $\mathrm{Si}$ & 7 & No \\
\hline 4- $26 \mathrm{M}$ & 9 & 12 & No & $10-12$ & 280 & $200-230$ & $\mathrm{RG}$ & No & $\mathrm{Si}$ & 8 & $\mathrm{Si}$ \\
\hline 5- $39 \mathrm{M}$ & 6 & 6 & No & 8 & 220 & $90-100$ & $\mathrm{RG}$ & $\mathrm{Si}$ & No & 6 & No \\
\hline $6-42 \mathrm{~N}$ & 4 & 5 & $\mathrm{Si}$ & 4- 6.5 & 220 & $80-120$ & $\mathrm{RG}$ & No & $\mathrm{Si}$ & 7 & No \\
\hline 7- $38 \mathrm{M}$ & 6 & 8 & No & $10-11$ & 280 & $150-220$ & RG & No & $\mathrm{Si}$ & 6 & No \\
\hline 8- $29 \mathrm{M}$ & 7 & 9 & SI & 7 & 240 & $170-200$ & $\mathrm{RM}$ & $\mathrm{Si}$ & No & 7 & $\mathrm{Si}$ \\
\hline 9- $37 \mathrm{M}$ & 5 & 7 & No & 6.5 & 220 & $100-90$ & RG & $\mathrm{Si}$ & No & 6.5 & No \\
\hline $10-38 \mathrm{M}$ & 11 & 12 & No & 10 & 280 & $230-250$ & $\mathrm{RG}$ & No & $\mathrm{Si}$ & 8 & $\mathrm{Si}$ \\
\hline $11-29 \mathrm{M}$ & 10 & 12 & No & $10-12$ & 280 & $200-230$ & $\mathrm{RG}$ & No & $\mathrm{Si}$ & 8 & $\mathrm{Si}$ \\
\hline $12-49 \mathrm{M}$ & 7 & 9 & No & 8 & 260 & $120-180$ & $\mathrm{RG}$ & No & $\mathrm{Si}$ & 7 & No \\
\hline $13-28 \mathrm{~N}$ & 10 & 12 & No & 6 & 220 & $80-80$ & RM & $\mathrm{Si}$ & No & 6 & No \\
\hline 14- $39 \mathrm{M}$ & 8 & 9 & No & 7 & 220 & $120-190$ & RM & $\mathrm{Si}$ & No & 7 & No \\
\hline $15-43 \mathrm{M}$ & 4 & 5 & No & 6.5 & 240 & $90-90$ & $\mathrm{RM}$ & $\mathrm{Si}$ & No & 6.5 & No \\
\hline $16-45 \mathrm{M}$ & 8 & 12 & No & 10 & 280 & $120-180$ & $\mathrm{RG}$ & No & $\mathrm{Si}$ & 7 & $\mathrm{Si}$ \\
\hline $17-25 \mathrm{M}$ & 4 & 6 & No & 7.5 & 280 & 80 & $\mathrm{RM}$ & $\mathrm{Si}$ & No & 7 & No \\
\hline $18-26 \mathrm{M}$ & 3 & 6 & No & 6.9 & 240 & $100-100$ & $\mathrm{RG}$ & $\mathrm{Si}$ & No & 6 & No \\
\hline $19-32 \mathrm{M}$ & 10 & 12 & No & 9 & 280 & $200-240$ & $\mathrm{RG}$ & No & $\mathrm{Si}$ & 7 & $\mathrm{Si}$ \\
\hline $20-36 \mathrm{M}$ & 8 & 9 & No & 7 & 260 & $180-220$ & $\mathrm{RG}$ & No & $\mathrm{Si}$ & 7 & No \\
\hline $21-41 \mathrm{M}$ & 7 & 11 & No & 9 & 260 & $200-240$ & RG & No & $\mathrm{Si}$ & 7 & $\mathrm{Si}$ \\
\hline $22-25 \mathrm{M}$ & 4 & 6 & No & 6 & 240 & $90-100$ & $\mathrm{RG}$ & $\mathrm{Si}$ & No & 6 & $\mathrm{Si}$ \\
\hline $23-45 \mathrm{M}$ & 6 & 6 & No & 7 & 260 & $120-100$ & RG & $\mathrm{Si}$ & No & 7 & $\mathrm{Si}$ \\
\hline 24- $34 \mathrm{M}$ & 6 & 9 & No & 7 & 240 & $180-200$ & $\mathrm{RG}$ & $\mathrm{Si}$ & No & 7 & No \\
\hline $25-38 \mathrm{M}$ & 6 & 7 & No & 6.5 & 240 & $200-230$ & $\mathrm{RG}$ & No & $\mathrm{Si}$ & 6.5 & No \\
\hline
\end{tabular}

M: Multípara; N: Nulípara; CAP: Complejo areola-Pezón; SSM: Surco submamario; TR: Tejido resecado en grs.; VC: Via de colocación; SG: Subglandular; RG: Retroglandular; RM: Retromuscular; SU: Suspensión Única; SD; Suspensión Doble; CC: Cirugía concomitante. 

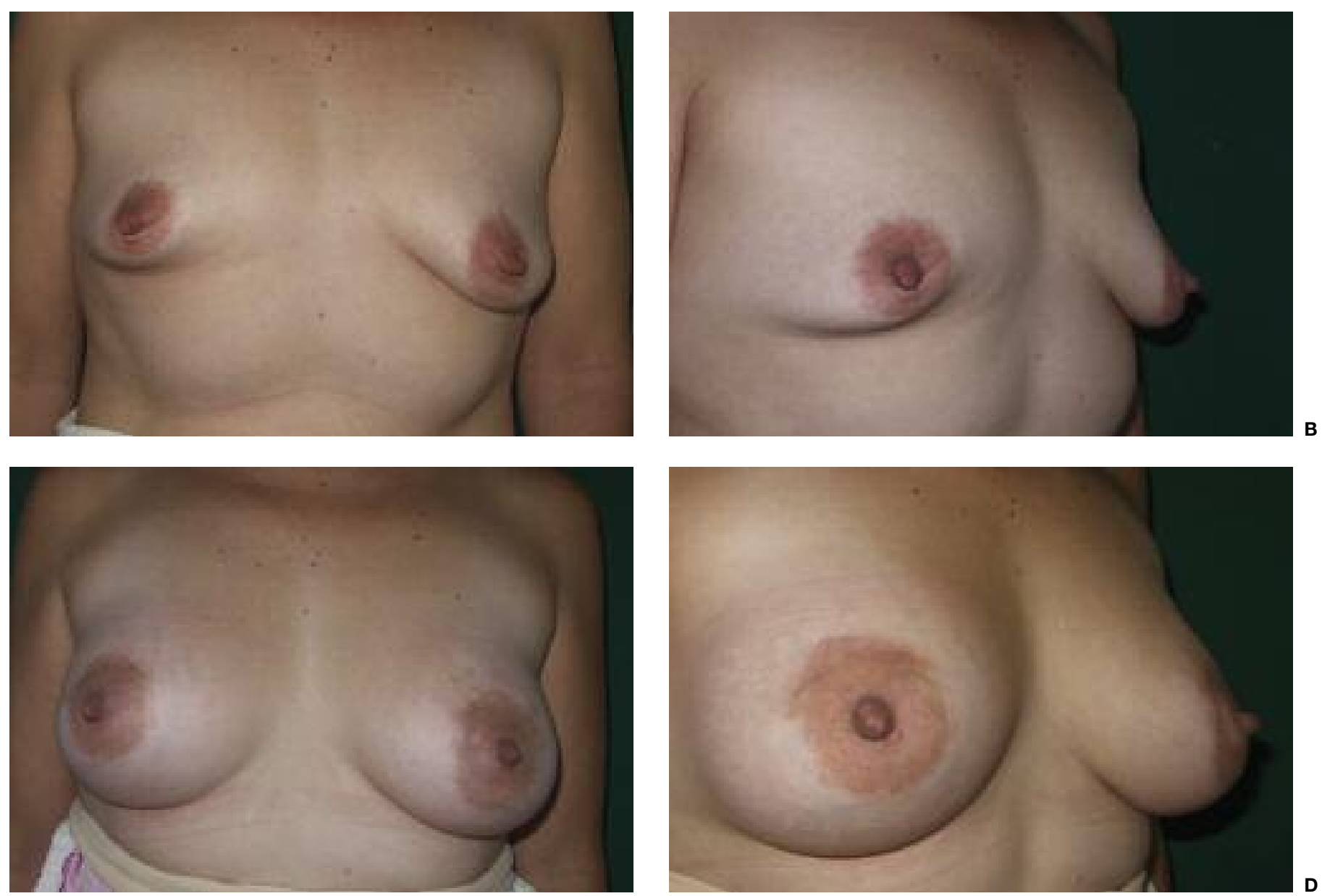

Fig. 3. A y B: Paciente multípara de 42 años con asimetría mamaria y mama tuberosa. Distancia areola-surco submamario derecha de $4 \mathrm{~cm}$ e izquierda de $6,5 \mathrm{~cm}$. Prevista elevación de $8 \mathrm{~cm}$ en mama izquierda y de $3 \mathrm{~cm}$ en la derecha, con corrección de la base de la mama en cruz, más colocación de una prótesis de 220 cc en lado derecho y de $180 \mathrm{cc}$ en lado izquierdo en plano retroglandular y doble fijación clavícula costilla. C y D: Postoperatorio a los 2 años sin recidiva. Extirpación de $\mathbf{8 0}$ gr en mama derecha y de 120 gr en mama izquierda. Distancia final areola-surco submamario de $7 \mathrm{~cm}$ en ambos lados.

cirugía se practicaron análisis preoperatorios y a quienes presentaron antecedentes heredofamiliares de carcinoma mamario se les practicó una mamografía. Se realizó seguimiento postoperatorio a los 15 días, 1 mes, 6 meses, 1 y 2 años de la intervención. Las piezas quirúrgicas extirpadas fueron sometidas a estudio anatomopatológico.

Marcaje: con la paciente en bipedestación (Fig. 2 A), se trazan las líneas que unen el punto medio clavicular (punto A), el pezón y el surco submamario (punto A') de cada mama y una línea transversal que une los puntos medios de cada brazo (B-B'). La intersección de las líneas A-A' y B-B', coincide con la proyección digital del surco submamario sobre la línea medio clavicular y será la nueva posición del pezón (punto E). Se mide el diámetro transverso de cada mama (línea C-D). Una vez realizados dichos trazos, se dibuja una media luna cuya parte más alta corresponde al punto $\mathrm{E}$, trazando una línea hacia ese punto que alcance ambos bordes supero-externos de la areola (Fig. 2 B).

Los implantes empleados en nuestras pacientes línea transversal de la mama, hiperproyectados, de perfil muy alto de 4 a $5 \mathrm{~cm}$, de gel cohesivo de alta densidad, texturizados, redondos, con volumen y peso mucho menor al volumen de la glándula mamaria original y dependiendo del tamaño del tórax y estatura de la paciente. Los volúmenes elegidos oscilaron entre los 180 y los $280 \mathrm{cc}$. Se colocaron implantes desiguales en caso de asimetría. Esta técnica sirve también para corregir mamas tuberosas (Fig. 3).

Utilizamos el plano retroglandular únicamente en mamas grandes y el subpectoral en mamas pequeñas. Con el fin de evitar recidivas, se utilizó la fijación del colgajo dérmico al periostio de la tercera costilla con sutura gruesa no reabsorbible Dermalon ${ }^{\circledR}$ 3-0 y únicamente si el complejo resultante de mama-implante resulta bastante pesado y voluminoso, colocamos un tirante interno de colgajo dérmico previamente fijado a la tercera costilla hacia arriba, hasta el periostio medio-clavicular mediante una aguja especial (Dr. Fuente del Campo) y siempre con material irreabsorbible del 3-0 a través de una incisión de $1 \mathrm{~cm}$. de ancho sobre la clavícula. 


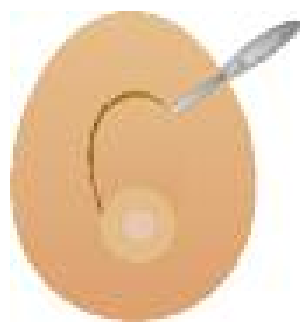

B

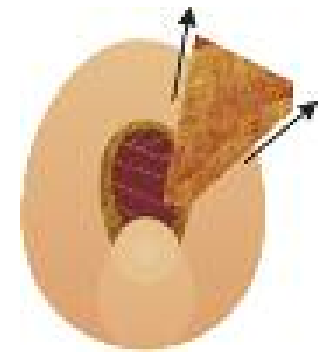

C

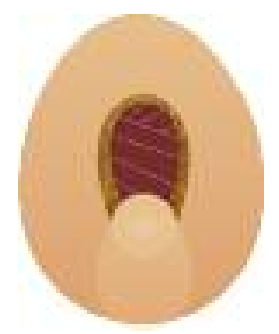

D

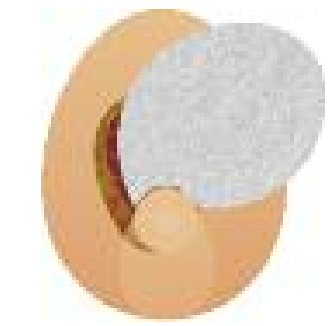

H

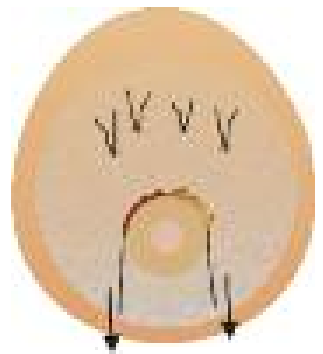

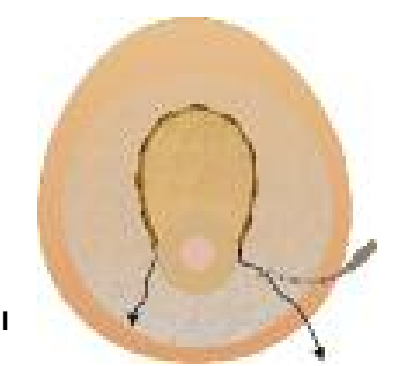

E

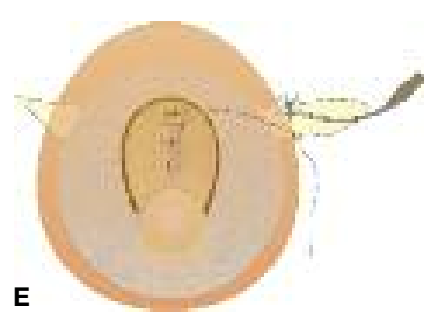

$\mathbf{F}$

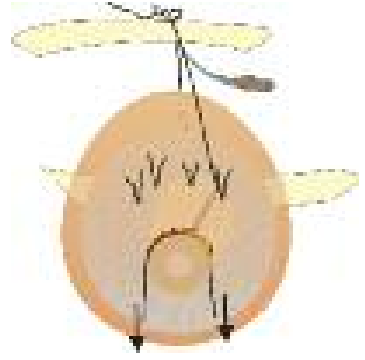

Fig. 4. a) Esquema de abordaje por vía supra-areolar semilunar. b) Retracción de colgajo dérmico hacia abajo y resección de pieza triangular de ambos cuadrantes superiores. c) Cavidad resultante y pilares laterales para introducción de prótesis. d) Introducción de prótesis de gel hiper-proyectada, texturizada, altamente cohesiva y de base poco menos ancha que el diámetro de la mama. e) Cierre sobre el implante de los colgajos superiores y fijación del colgajo dérmico a la tercera costilla. f) Si el complejo mama-implante resulta demasiado grande y pesado, se realiza nueva pexia, además de la clavicular en la tercera costilla ipsilateral. g) Aguja de Reverdin para fijación clavicular. h) Fijación de colgajo supra-areolar con 3 ó 4 puntos de tensión mínima sobre porciones de silicona blanda con 3 propósitos : 1) Evitar que la cicatriz resultante quede ancha. 2) Evitar distorsión de la areola. 3) Evitar la lesión de la piel subyacente. h) Cierre en bolsa de tabaco.

\section{Técnica quirúrgica}

Realizamos la intervención bajo anestesia general mediante mascarilla faríngea y con la colaboración de dos ayudantes.

Infiltramos solución de Klein en ambas mamas. Si la distancia desde el punto infra-areolar al surco submamario mide más de $7 \mathrm{~cm}$, realizamos liposucción inframamaria a través de una incisión central de $1 \mathrm{~cm}$ de ancho colocada sobre el nuevo surco submamario en la zona donde se van a dejar los drenajes finales, con el fin de formar un nuevo surco submamario mediante la colocación de 3 ó 4 presillas a cada lado de dicha incisión, que van de la piel al periostio costal. Comenzamos desepitelizando ambas medias lunas mediante torniquete basal en la forma habitual (Fig. 4 A). Posteriormente realizamos un corte a medio $\mathrm{cm}$. del borde inferior de la elipse y levantamos el colgajo dérmico hasta $2 \mathrm{~cm}$ antes del borde superior areolar, evertiéndolo hacia el pezón. Después, extirpamos parte de ambos cuadrantes superiores de la mama, con la ayuda de un bisturí eléctrico, llegando en profundidad hasta la fascia muscular (Fig. 4 B). Si el plano elegido fue el submuscular, desde ahí mismo seccionamos el pectoral mayor transversalmente y en forma

de L invertida siguiendo el borde esternal de inserción de dicho músculo. De no ser submuscular, disecamos subglandularmente por abajo hasta el surco submamario y 1 ó $2 \mathrm{~cm}$ por debajo de éste, para conseguir una distancia de 6 a $7 \mathrm{~cm}$ desde el reborde areolar hasta el nuevo surco submamario, siguiendo una disección más hacia la línea media que hacia los lados para evitar que los implantes se lateralicen (Fig. 4 C). Con la ayuda de separadores, procedemos a colocar los implantes (Fig. 4 D), consiguiendo cerrar los bordes glandulares sobre los mismos, desde la areola hasta la fascia; empleamos Monocril @ 3-0, previa cuidadosa hemostasia. De ser posible, no utilizamos drenajes cuando la colocación de los implantes fue retroglandular; por el contrario, los utilizamos por salida axilar cuando la colocación fue retromuscular. El cierre glandular debe ser perfecto para evitar herniación de la prótesis y conseguir además la conización de la mama y la reducción de su anchura y volumen (Fig. 4 E).

A continuación, fijamos el colgajo dérmico mediante sutura irreabsorbible de 3-0 en el periostio de la tercera costilla; este tipo de fijación la empleamos en mamas grandes y pequeñas. Si el colgajo dér- 

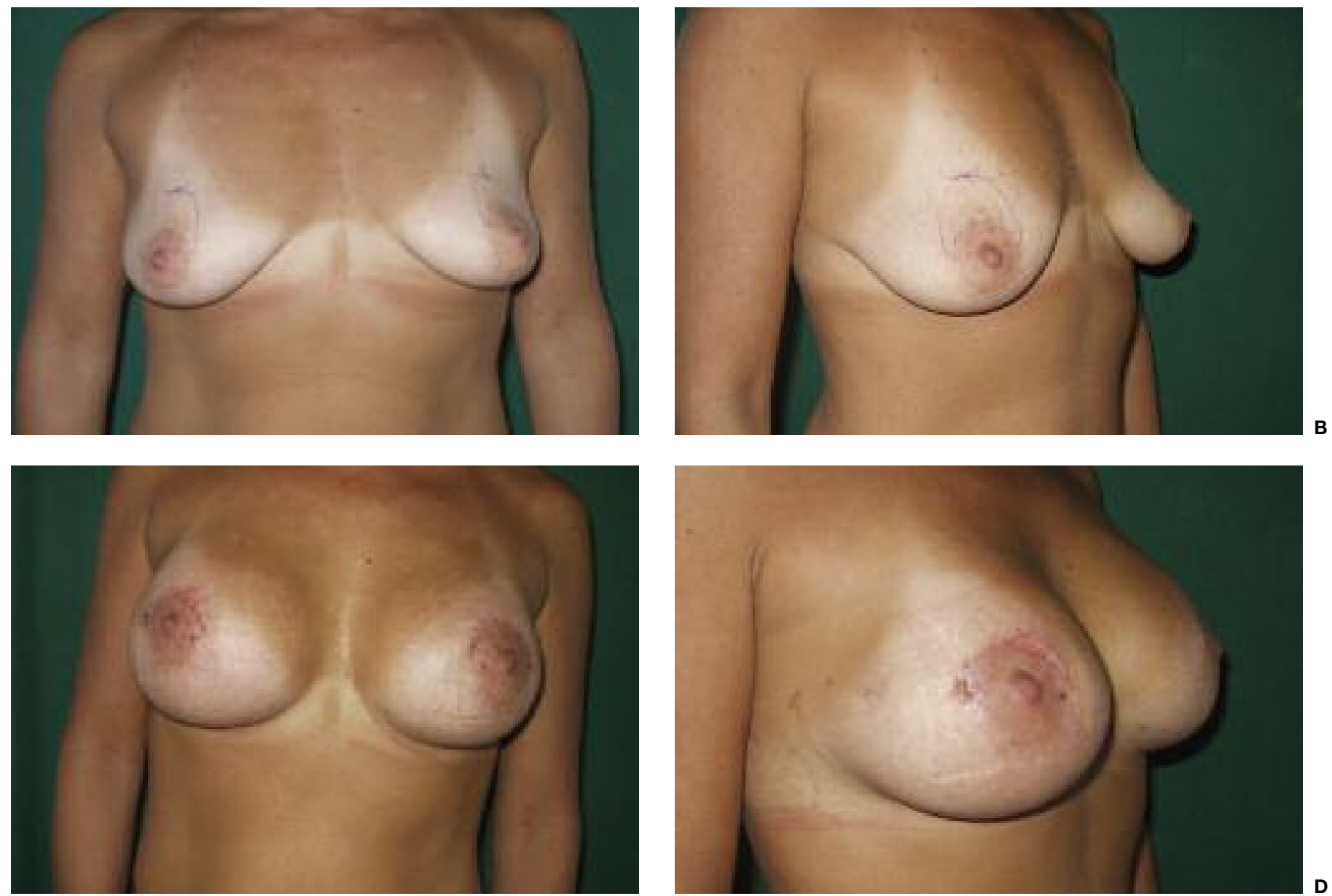

Fig. 5. A-B: Paciente multípara de 26 años. Elevación prevista de $7 \mathrm{~cm}$ en mama izquierda y de $6 \mathrm{~cm}$ en la derecha. Plano de los implantes retromuscular con fijación única del colgajo dérmico a la costilla. Implantes de gel cohesivo preproyectados de $9 \mathrm{~cm}$ de diámetro y $260 \mathrm{cc}$ de volumen. C-D: Postoperatorio temprano a los 30 días. Se extirparon $220 \mathrm{gr}$ de mama izquierda y $100 \mathrm{gr}$ de la derecha. Distancia final areola-surco submamario de $7 \mathrm{~cm}$.

mico resulta corto podría distorsionar la areola, en cuyo caso lo elongamos mediante una zeta-plastia que se practica con la paciente sentada en la mesa de operaciones. El colgajo dérmico se expande mediante puntos sueltos de Monocril @ 3-0 sobre el cierre glandular, para proteger y reforzar la nueva forma de la glándula sobre la prótesis. Hecho esto, procedemos, si el complejo mama-implante resulta muy pesado y grande, a colocar en la porción más distal del colgajo dérmico, a $2 \mathrm{~cm}$. de la fijación del mismo sobre la costilla (Fig. 4 F), un tirante de sutura irreabsorbible de Nylon 3-0 mediante la ayuda de una aguja de Reverdin (Fig. $4 \mathrm{G}$ ) hacia la línea medio-clavicular, que se fijó en el periostio hemiclavicular ,teniendo la precaución de hacerlo precisamente sobre la marca transversal preoperatoria A, porque de otra manera la incisión sería visible, debido a la variación de las marcas con la posición en decúbito de la paciente. Se sugiere, antes de realizar la fijación hemiclavicular, colocar a la paciente en posición de semiflexión con los tirantes dobles no muy tensos, sino relajados.

El siguiente paso consiste en cerrar la piel; para evitar la tensión ejercida por el peso de las mamas y de las prótesis sobre la línea de sutura periareolar se colocan 4 ó 5 puntos de relajación $5 \mathrm{~cm}$ más allá del cierre periareolar con Nylon 3-0 y aguja grande curva cortante del número 10, entrando por piel (colgajo superior), atravesando todo el espesor del colgajo superior, y por abajo, fijándose sobre el colgajo dérmico para salir de nuevo por donde se penetró la piel del colgajo superior. Con el objeto de evitar el sufrimiento de la piel y la cicatrización indeseable, la sutura se fija sobre una pequeña porción de silicona blanda $0.5 \mathrm{~cm}^{3}$, de la utilizada habitualmente para los implantes de mentón (Fig. 4 H). Nos ayudamos en esta tarea del estiramiento previo, por parte del ayudante, del colgajo superior a la areola mediante dos ganchos dobles hacia el pezón. Hecho todo esto, la sutura periareolar quedará sin tensión para su cierre en tres capas. La primera capa se cierra en forma de bolsa de tabaco, con sutura subdérmica de Prolene ${ }^{\circledR}$ 3-0 y aguja recta (Fig. 4 I). Para cerrar la segunda capa hacemos pequeños cortes de relajación a $1 \mathrm{~mm}$ del reborde periareolar, en la base del colgajo dérmico, buscando evertir el borde areolar; empleamos puntos separados de Monocril ${ }^{\circledR}$ 5-0, tratando de aco- 

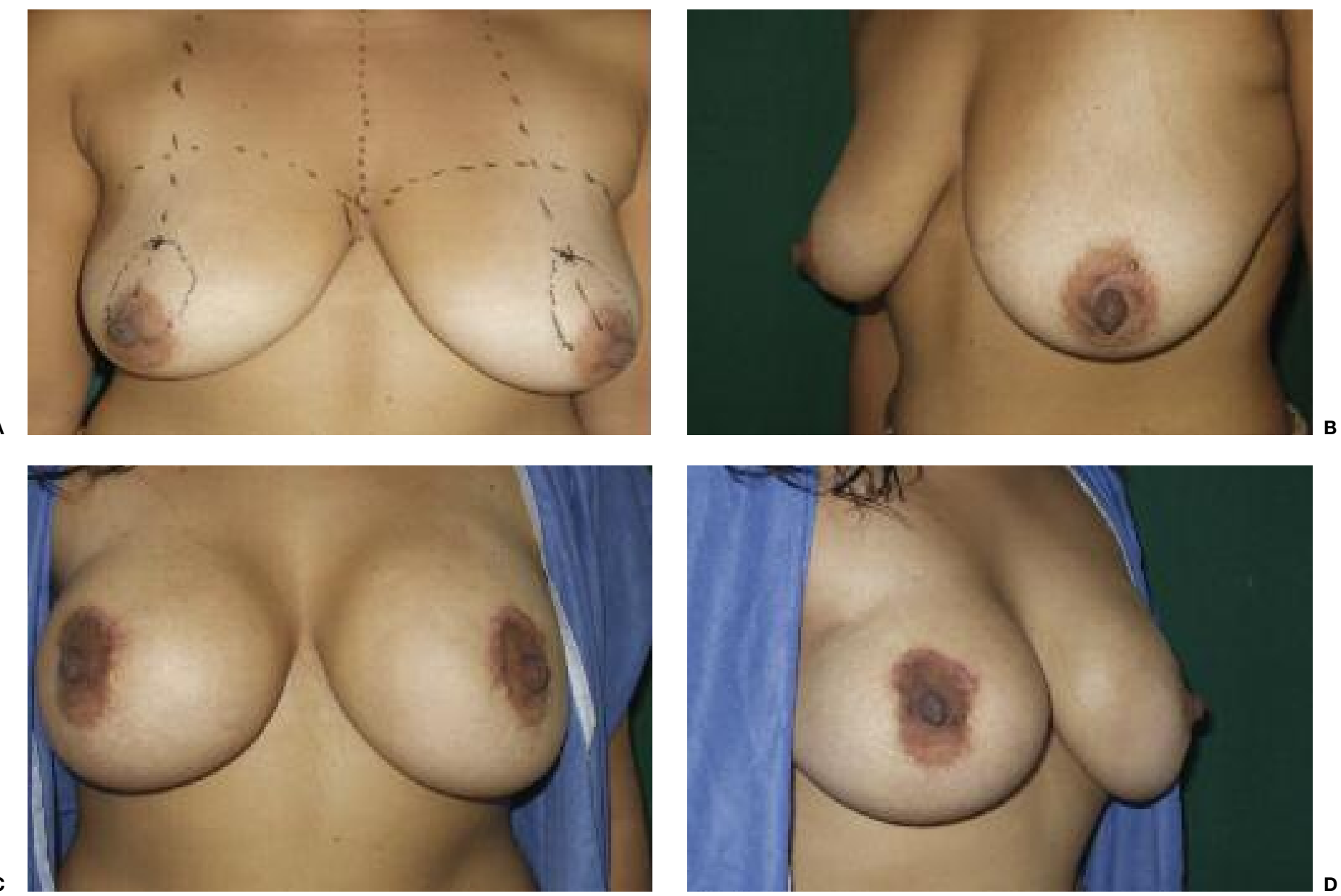

Fig. 6. A-B: Paciente de 29 años, multípara (2 partos). Programada para mastopexia de aumento más liosucción de axilas y surcos submamarios. Elevación prevista de $11 \mathrm{~cm}$ en el lado derecho y de $12 \mathrm{~cm}$ en el izquierdo. Distancia areola-surco submamario de $12 \mathrm{~cm}$ en mama izquierda y de $10 \mathrm{~cm}$ en la derecha. Colocación de implantes de gel cohesivo hiperproyectados de 280 cc de volumen y con base más estrecha que el diámetro mamario, en plano retroglandular. Doble fijación costilla y clavícula. C-D: Postoperatorio tardío al año. Extirpación de 230 gr de mama izquierda y $200 \mathrm{gr}$ de la derecha. Distancia areola-surco submamario final de $8 \mathrm{~cm}$.

modar y ajustar los bordes de sutura. La última capa se cierra con puntos continuos en U de Prolene ${ }^{\circledR} 5$ 0 . Por ultimo, sentamos a la paciente para observar la simetría. El tiempo operatorio total osciló entre los 90 y los 120 minutos para ambas mamas

Aconsejamos colocar al final Micropore ${ }^{\circledR}$ de $6 \mathrm{~cm}$ de ancho en forma de $\mathrm{U}$ alrededor de ambas mamas para evitar tensión sobre las suturas y mantenerlo más de 10 días, pudiendo la paciente asearse desde el día siguiente con jabón antiséptico y cambiando únicamente la cura colocada sobre la herida periareolar diariamente. Es importante el uso de un vendaje almohadillado con Reston ${ }^{\circledR}$ (variedad de espuma de poliuretano) y el uso de una banda estabilizadora sobre el nuevo surco submamario El sujetador indicado es el de reconstrucción mamaria con velcro anterior y prolongación torácica inferior de preferencia de lycra.

\section{Resultados}

La elevación conseguida de los pezones osciló entre los $5 \mathrm{~cm}$ en el menor de los casos, hasta los 10$12 \mathrm{~cm}$ en grandes ptosis. Más de 2 años después, no ha habido ninguna recidiva (Fig. 5 y 6). La simplicidad de la técnica permite su combinación con otras intervenciones como liposucción, dermolipectomía, etc (Fig. 7). En el $90 \%$ de los casos la cirugía fue ambulatoria; únicamente en cirugía combinada la paciente pasó 24 hrs. en el hospital.

El postoperatorio es prácticamente indoloro, excepto cuando los implantes se colocan en el plano retromuscular. La paciente puede efectuar ejercicio a los 15 días de la intervención, siempre y cuando tenga el sujetador colocado. Las cicatrices evolucionaron satisfactoriamente, incluso en pacientes de piel oscura; cuando fue necesario se colocaron placas de silicona a los 15 días sobre las cicatrices resultantes y se mantuvieron durante un mes. No hubo necesidad de masajes.

En el capítulo de complicaciones, sufrimos durante la curva de aprendizaje de la técnica una dehiscencia parcial de la sutura en un lado y en otra paciente una dehiscencia parcial de ambas heridas que ameritó corrección con anestesia local. Dos de las pacientes con el tirante clavicular presentaron tirantez y en otras 2 apareció ardor que cedió con la inyección tópica de 


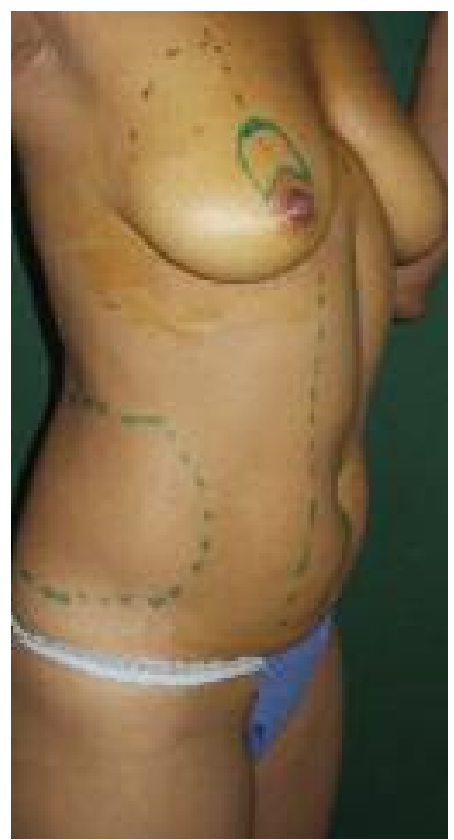

A

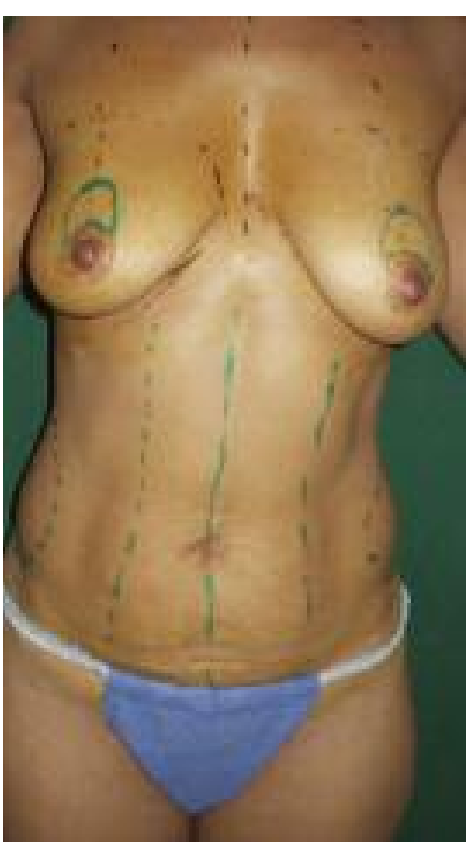

B

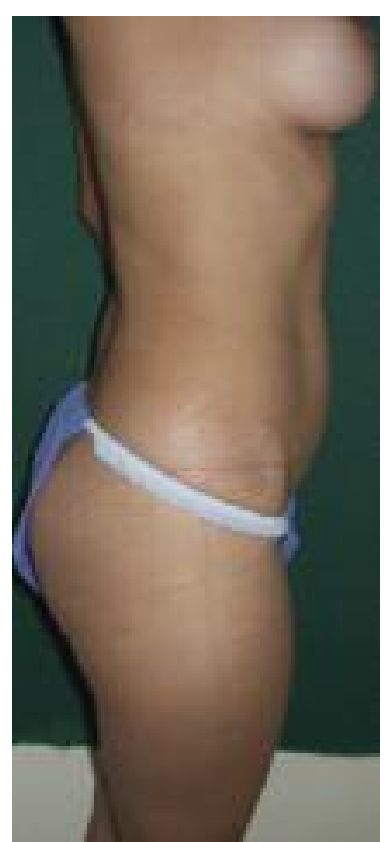

C

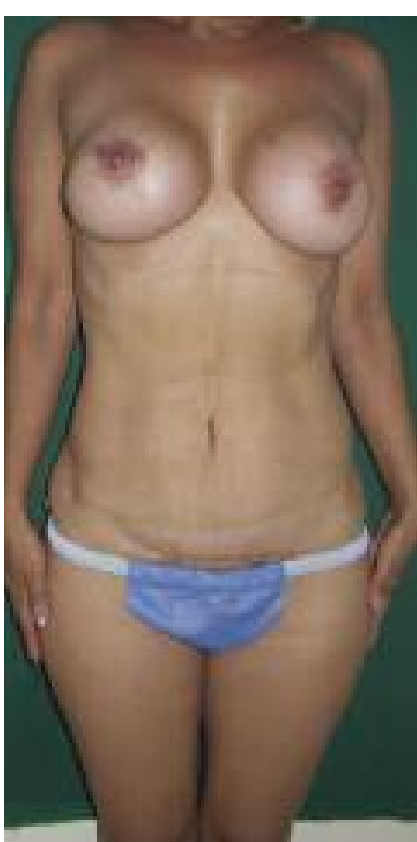

D

Fig. 7. A-B: Paciente de 45 años, multípara (3 cesáreas), programada para mastopexia de aumento, dermolipectomía abdominal por miniabdominplastia, plicatura de rectos, desinserción de la base del pedículo umbilical y nueva fijación más liposucción abdominal, de caderas y de flancos. Elevación mamaria prevista de $6 \mathrm{~cm}$ en cada lado. Implantes de gel cohesivo hiperproyectados de base menor que el diámetro mamario y 260 cc de volumen, plano retroglandular. Fijación únicamente a costilla. C-D: Postoperatorio a los 6 meses. Extirpación de 120 gr en mama izquierda y $100 \mathrm{gr}$ en la derecha. Distancia final areola-surco submamario de $7 \mathrm{~cm}$.

0,3 cc de Triamcinolona; estos últimos fueron casos en los que la elevación del pezón alcanzó los $12 \mathrm{~cm}$. No hubo parestesias, endurecimiento o desplazamiento de los implantes en ninguno de los controles. Ninguna paciente presentó extrusiones, sepsis, infecciones, o hematomas. La elongación discreta de la areola es una de las complicaciones menores que amerita corrección secundaria posterior (Fig. 8). Sin embargo en areolas pequeñas, esto no sucede, o es mínimo (Tabla II).

Lo que mas les gustó a las pacientes fue la mejoría del hemisferio superior de la mama, así como la centralización y mejoría del surco intermamario.

En cuanto a los resultados del estudio anatomopa- tológico de las piezas de resección, fueron de mastopatía fibroquística en el $80 \%$ de los casos y únicamente se reportó papilomatosis múltiple intraductal en un caso de recambio de implantes (Fig. 9) que se trató con 3 dosis de vacuna contra el virus del papiloma humano y seguimiento por su ginecólogo; en otros 2 casos se informó de ectasia ductal.

\section{Discusión}

Aunque es muy poca la casuística que presentamos y muy corto el tiempo de seguimiento, los resultados obtenidos con nuestra técnica creemos que han sido muy satisfactorios.

Tabla II.

Elevación de los pezones

Cirugía ambulatoria

Estancia hospitalaria de $24 \mathrm{~h} *$

*Cuando hay cirugía combinada y no por la mastopexia en si

Tiempo para volver a realizar ejercicio

Cicatrización

Volumen colocado de implantes

Pseudo-Ptosis Postoperatoria

Característica que prefirió el paciente
$5-12 \mathrm{~cm}$

$90 \%$

$10 \%$

15 días

Altamente satisfactoria

Entre los 180 a los $300 \mathrm{cc}$

Ninguna

Mejoría del hemisferio superior

Centralización

Mejoría del surco ínter mamario

Dehiscencia parcial en 2 pacientes.

Sensación de tirantez y ardor por el tirante clavicular

Elongación discreta de la areola en levantamientos $>12 \mathrm{~cm}$

Ligera deformación de areola superior en los primeros casos 


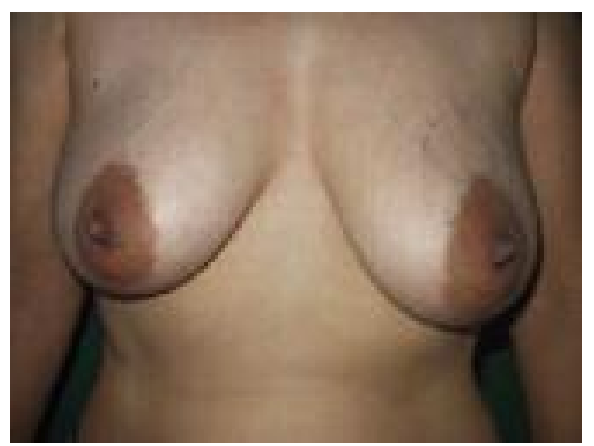

A

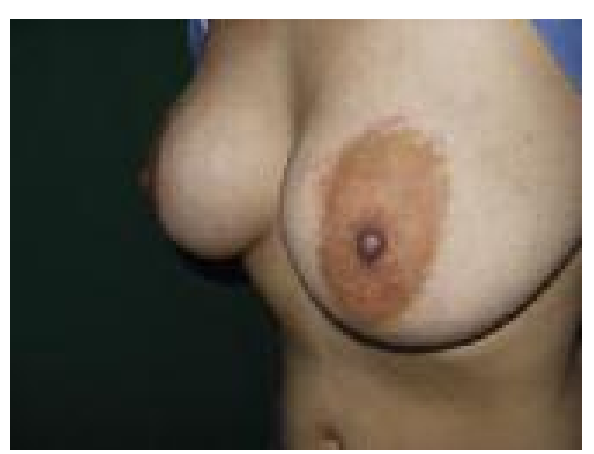

D

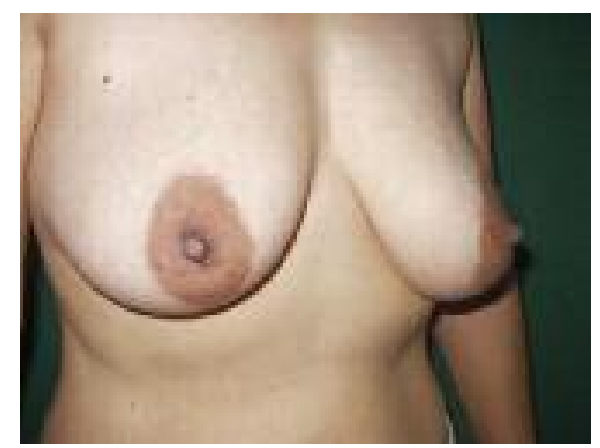

B

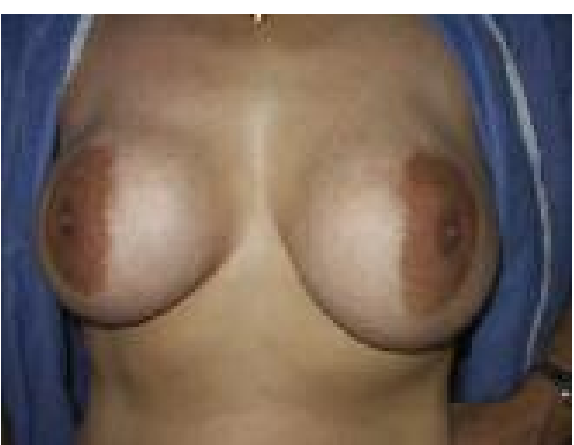

E

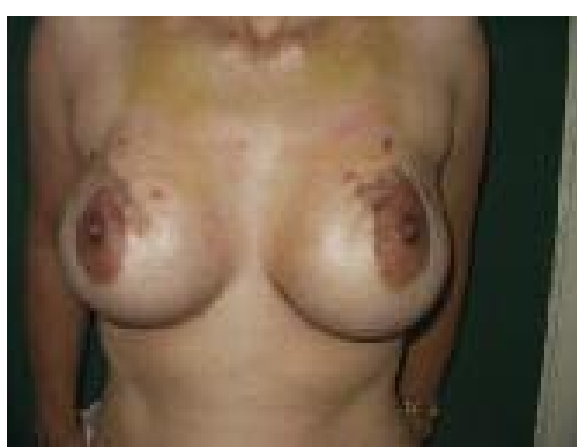

C

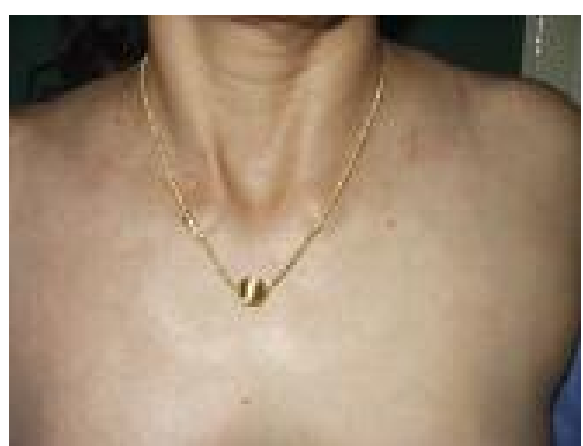

F

Fig. 8. A: Paciente multípara de 32 años. Programada para elevación de $12 \mathrm{~cm}$ en mama izquierda y $10 \mathrm{~cm}$ en la derecha, más liposucción submamaria y axilar. Distancia areola-surco submamario de $9 \mathrm{~cm}$. Implantes texturizados de $280 \mathrm{cc}$ en plano retroglandular. Doble fijación costilla y clavícula. B: Preoperatorio, vista de tres cuartos. C: Postoperatorio a los 10 días. Extirpación de 240 gr en mama izquierda y 200 gr en la derecha. Distancia final areola-surco submamario de $7 \mathrm{~cm}$. D: Postoperatorio a los 6 meses con ligera elongación de la areola. E: Postoperatorio al año. F: Imagen acercada de la cicatriz hemiclavicular, imperceptible a los 6 meses de la intervención.

No intervenimos pacientes con mamas hipertróficas grados III y IV, sino únicamente las mamas ptósicas y con falta de volumen. Aunque los trabajos de

Frey (31) en 1999, mencionan el colgajo dérmico, este autor lo utiliza como sustento para que la cicatriz no se ensanche, pero no maneja la fijación costal. Si
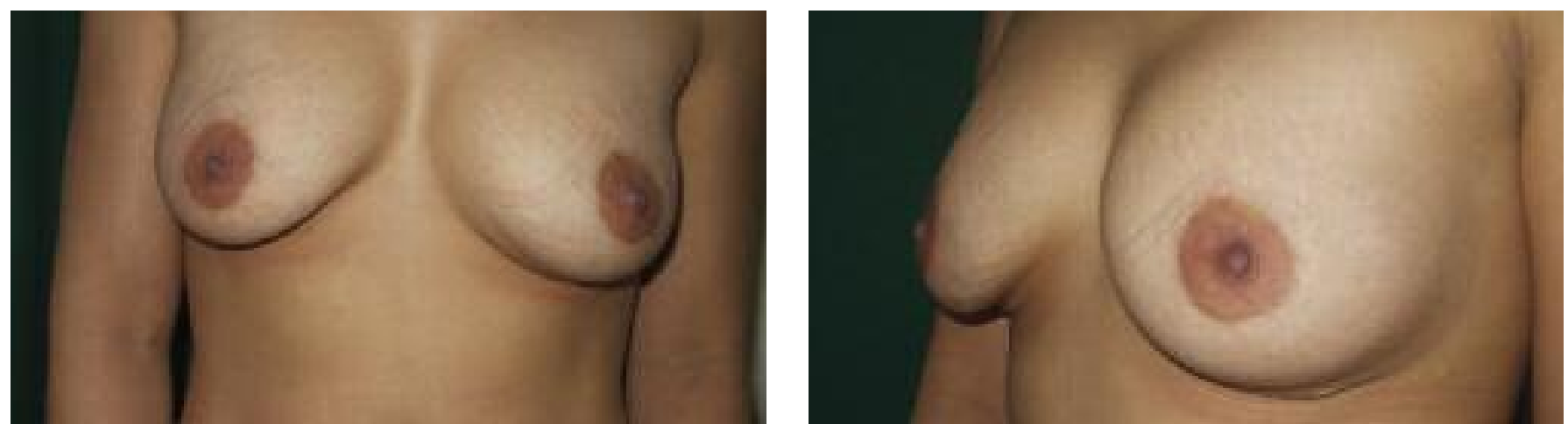

C
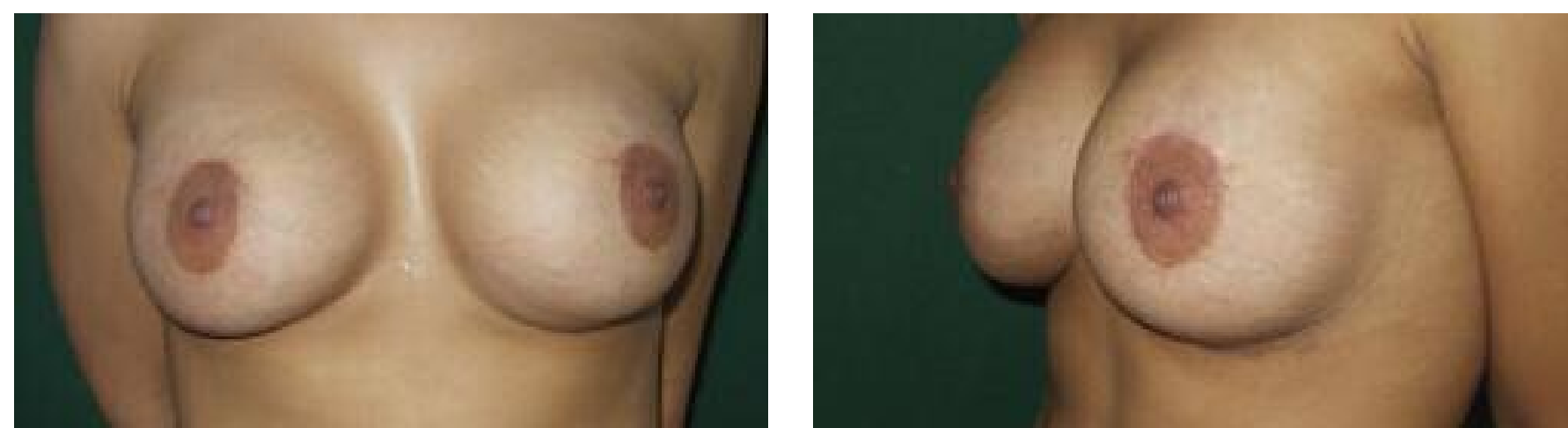

Fig. 9. A-B: Paciente de 24 años, multípara (2 partos), con implantes mamarios de $350 \mathrm{cc}$ de 10 años de antigüedad, programada para recambio de implantes en plano retromuscular y capsulectomía bilateral con nuestra técnica. Distancia areola-surco submamario de $7,5 \mathrm{~cm}$. Elevación prevista de la mama de $4 \mathrm{~cm}$ en el lado derecho y de $6 \mathrm{~cm}$ en el izquierdo. Colocación de nuevos implantes de $280 \mathrm{cc}$ y base menor que el diámetro mamario previo. Fijación única a costilla. C-D: Postoperatorio al año. Extirpación de $80 \mathrm{gr}$ en ambos lados. Distancia final areolasurco submamario de $7 \mathrm{~cm}$. 
comparamos nuestra técnica con los importantes trabajos de Fellicio (17) y Benelli (18) de 1991, basados en una técnica periareolar que sirvió de base a nuestro trabajo, observamos que quedaban unas mamas muy planas, casi sin proyección y con una alta posibilidad de recidiva de la ptosis, además de con una cicatriz grande periareolar en personas de piel oscura y una disminución de la sensibilidad del pezón.

Con respecto al trabajo de Sampaio (19), podemos decir que esta técnica presenta unos resultados muy buenos, pero con el inconveniente de usar malla sintética, lo cual nos podría distorsionar una mamografía, además de añadir una mayor susceptibilidad a infección.

Revisando el reciente trabajo de Cárdenas y Ramírez Macías (29), vemos que estos autores hacen una minuciosa revisión de las indicaciones precisas actuales, con una casuística abundante, pero sin embargo su técnica provoca cicatrices periareolares y verticales y no incorpora una aparente fijación de la glándula al periostio.

Nosotros, en 2 de nuestros casos en lo que la distancia areola-surco submamario rebasaba $\operatorname{los} 7 \mathrm{~cm}$, creamos un nuevo surco submamario mediante liposucción calculando finalmente una distancia areolasurco submamario de 6 a $7 \mathrm{~cm}$.

En la técnica descrita por Ceydeli (27), la movilización y desprendimiento de toda la glándula resulta un tanto arriesgada, sobre todo al efectuar un corte en la parte inferior, además de ser una técnica periareolar que podría afectar a la futura sensibilidad.

Gulyas (23) presenta ya el colgajo dérmico, sin embargo su técnica conlleva un despegamiento grande y una cicatriz periareolar además de otra vertical.

Insistimos mucho en el tipo de implante, que de preferencia será texturizado, de perfil alto, de cohesividad máxima y lo más importante, con un diámetro que sea 1 ó $2 \mathrm{~cm}$ menor que el diámetro real de la mama; recordemos que buscamos turgencia y proyección, no volumen ni peso.

También observamos la nula aparición de ptosis mamaria recurrente a largo plazo entre nuestras pacientes. Finalmente señalar que el diámetro de la areola puede reducirse después al gusto de las pacientes.

\section{Conclusiones}

Proponemos una alternativa más en la cirugía de corrección de la ptosis mamaria mediante una técnica que acorta el tiempo operatorio, minimiza la cicatriz y ofrece mejores resultados a largo plazo. También permite la corrección de otro tipo de problemas como mamas tuberosas, asimetría mamaria e hipertrofia mamaria virginal.
Además, al extirpar los cuadrantes superiores mamarios disminuye la probabilidad, dada la edad de nuestras pacientes, del temible carcinoma mamario abriéndonos la posibilidad cuando el caso así lo requiera por antecedentes heredofamiliares, de practicar una cirugía profiláctica con resultados predecibles por su seguridad, versatilidad y facilidad de ejecución.

\section{Agradecimientos}

Agradecemos la valiosa ayuda prestada para la elaboración de este trabajo por los licenciados Erika Barnard en diseño gráfico y Rubén Díaz en informática.

\section{Dirección del autor}

\section{Dr. Ramón Navarro Ceballos}

Calle 54 No. 364 T por 33A Edificio CMA Departamento 102, Mérida, Yucatán, México. CP: 97000

Apartado Postal 4-30

e-mail: cemay@prodigy.net.mx

\section{Bibliografía}

1. Wiesenberger H.: "Blutversorgung und zirkuläre Umschnedung des Warzenhofes". Zentralbl f Chir 1928; 55: 2385.

2. Thorek M.: "Plastic Surgery of the breast and abdominal Wall". American Journal of American Sciences. 1942; 204 (6): 887.

3. Passot R.: "La correction du prolapsus mammaire par le procede de la transposition du mamelon". La presse medicale 1925; 33: 19.

4. Strömbeck J O.: "Mamaplasty: report of new technique based on the two pedicles procedure". Br. J. Plast Surg. 1960; 13: 79.

5. Aufricht J.: "Mamaplasty for pendolous breasts empiric and geometric planning". Plast. Reconstr. Surg. 1949; 4: 13.

6. Noel A.: "La Chirugie Estetique et son role social Paris". Masson et Cie 1926.

7. Mckissock P K.: "Reduction mamaplasty with a vertical Dermal flap". Plast. Reconstr. Surg, 1972; 49(3): 245.

8. Goulian D.: "Dermal Mastopexy". Plast. Reconstr. Surg, 1971;47(2):105

9. Perras C.: "The creation of a twin Breast Following Radical Mastectomy". Plast. Reconstr. Surg. 1978; 61(3): 467.

10. Regnault P C.: "Indications for breast augmentations". Plast. Reconstr. Surg. 1967; 40: 524.

11. Hinderer M.: "Cirugía mamaria de aumento y reducción". Ann. Acad. Med. Quir. Esp. 1965; 3: 50.

12. Arie G.: "A new technique of Mamaplasty". Plast. Reconstr. Surg. and transplantation bulletin. 1958; 21(2): 159.

13. Pitanguy.: "Mamaplastias estudio de 245 casos consecutivos e apresentaçao de técnica pessoal". Rev. Bra. Cirurg. 1961; 42: 201.

14. Peixoto G.: "Reduction Mamoplasty: A personal technique". Plast. Reconstr. Surg. 1980; 62(2): 217.

15. Ribeiro L.: "A new technique for reduction mamaplasty". Plast. Reconstr. Surg. 1975; 55: 330.

16. Pontes R.: "Single stage reconstruction of the missing breast". Br. J. Plast. Surg. 1976; 26: 377.

17. Fellicio Y.: "Periareolar. Reduction Mamaplasty". Plast. Reconstr. Surg. 1991; 88(5): 789.

18. Benelli L.: "A new periareolar mammaplasty: the round block, technique Aesthetic". Plast. Surg. 1990; 14 (2): 93.

19. Góes J C S.: "Periareolar Mamaplasty: Double Skin Technique". Plast. Reconstr. Surg. 1991; 87(3): 595.

20. Orlando J, Guthrie R. Jr.: "The superomedial dermal pedicle for nipple transposition" Br. J. Plast. Surg. 1975; 28(1): 42.

21. Rappaport N, Spira M.: "A simplified technique for deepithelial- 
ization in patients requiring augmentation mastopexy". Plast. Reconstr. Surg. 1982; 70(4):4.

22. Puckett C, Meyer V.: "Crescent mastopexy and augmentation". Plast. Reconstr. Surg. 1985; 75(4): 533.

23. Gulyas G.: "Mammaplasty with a periareolar dermal cloak for glandular support Aesthetic". Plast. Surg. 1999; 23(3): 164.

24. Kirwan L.: "Augmentation of the ptotic Breast: Simultaneous Periareolar Mastopexy/Breast Augmentation". Aesth. Surg. J. V. 1999; 19(1):34.

25. Karnes J, Morrison W.: "Simultaneous breast augmentation and lift". Aesthetic Plast. Surg. 2000; 24(2): 148.

26. Graf R, Biggs T.: "In search of better shape in mastopexy and reduction mammoplasty". Plast. Reconstr. Surg. 2002; 110(1): 309.

27. Ceydeli A, Freund R.: "Tear-drop augmentation mastopexy: a technique to augment superior pole hollow". Aesthetic Plast. Surg. 2003; 27(6): 425 .

28. Auersvald A.: "Extended crescent mastopexy with augmentation". Aesthetic Plast. Surg. 2006; 30(3): 275.

29. Cárdenas-Camarena $L$, Ramirez-Macias R.: "Augmentation/mastopexy: how to select and perform the proper technique". Aesthetic Plast Surg. 2006; 30(1):21.

30. De la Fuente A, Martin del Yerro J.: "Periareolar mastopexy with mammary implants". Aesthetic Plast. Surg. 1992; 16(4): 337.

31. Frey M.: "A new technique of reduction mammaplasty: dermis suspension and elimination of medial scars". Br. J. Plast. Surg. 1999; 52(1): 45.

32. Gasperoni C, Salgarello M.: "Experience and technical refinements in the "donut" mastopexy with augmentation mammaplasty". Aesthetic Plast. Surg. 1988; 12(2):111.
33. Holmstrom H, Lossing C.: "Reduction mammaplasty with a sliding nipple technique". Scand J. Plast. Reconstr. Surg. Hand Surg. 1990; 24(3): 245.

34. Lewis G.: "A method of mastopexy with fascia lata transplants". J. Int. Coll. Surg. 1956; 26(3): 346.

35. Weiner D, Aiache A, Silver L, Tittiranonda T.: "A single dermal pedicle for nipple transposition in subcutaneous mastectomy, reduction mammaplasty, or mastopexy". Plast. Reconstr. Surg. 1973; 51(2): 115 .

36. Andrews J , Yshizuki M, Martins D , Ramos R.: "An areolar approach to reduction mamaplasty". Br. J. Plast. Surg. 1975; 28(3): 166.

37. Bartels R , Strickland D, Douglas W.: "A new mastopexy operation for mild or moderate breast ptosis”. Plast. Reconstr. Surg. 1976; 57(6): 687.

38. Auclair E, Mitz V.: "Repair of mammary ptosis by insertion of an internal absorbable support and periareolar scar". Ann. Chir. Plast. Esthet. 1993; 8(1): 107.

39. Brink R.: "Management of true ptosis of the breast ". Plast. Reconstr. Surg. 1993; 91(4): 657.

40. Kuzbari R, Deutinger M, Todoroff B P, Schneider B, Freilinger G.: "Surgical treatment of developmental asymmetry of the breast. Long term results". Scand J. Plast.Reconstr. Surg. Hand Surg. 1993; 27(3): 203.

41. Marconi F, Cavina C.: "Reduction mammaplasty and correction of ptosis: a personal technique". Plast. Reconstr. Surg.1993; 91(6): 1046. 